\title{
Manejo médico de la miomatosis uterina. Revisión de la literatura
}

\author{
Janer Sepúlveda A. ${ }^{1}$ Miguel Ángel Alarcón $N^{2}$ \\ ${ }^{1}$ Departamento de Gineco-Obstetricia, Profesor Asociado, Director del Grupo de Investigación GINO, ${ }^{2}$ Departamento de \\ Gineco-Obstetricia, Profesor Titular. Universidad Industrial de Santander. Bucaramanga, Colombia.
}

\section{RESUMEN}

Objetivo: Proporcionar un enfoque basado en la mejor evidencia disponible de las diferentes opciones de tratamiento médico para los miomas uterinos. Método: Se realizó una búsqueda bibliográfica en las bases de datos, Med Line PubMed, Embase, Cochrane, Ovid- Hinari, Scielo, Bireme y Lilacs. Resultados: Se revisaron 212 artículos y se seleccionaron 58, los cuales estaban relacionados directamente con el tema. Hay múltiples tratamientos que han sido usados para el tratamiento de miomatosis. Los agonistas de la $\mathrm{GnRH}$, son los únicos medicamentos aprobados por la Food and Drug Administration, para la disminución del volumen de los miomas, usados pre-quirúrgicamente, sin embargo presentan muchos efectos secundarios. El dispositivo liberador de levonogestrel se plantea como una opción para pacientes con miomas menores de 6 centímetros que no afecten la cavidad uterina. Los moduladores selectivos de receptores de progestágeno y los inhibidores de aromatasa se plantean como un manejo que puede ser útil, especialmente para pacientes en la perimenopausia. Conclusión: La literatura muestra que hay evidencia de varios medicamentos que pueden ser usados para el manejo de pacientes con miomatosis, especialmente para el manejo de los síntomas asociados para mejorar su calidad de vida y las pacientes que obtienen mayor beneficio son las que están cercanas a la menopausia. Finalmente hay otros fármacos que son objeto de investigación y pueden ser futuras opciones terapéuticas para su uso en conjunto tanto en la miomectomía y como uso exclusivo de tratamiento médico.

\section{PALABRAS CLAVE: Manejo médico, mioma}

\section{SUMMARY}

Objective: We offer an approach of scientific literature based on the best available evidence of the different medical treatment options for uterine fibroids. Method: A search of the literature in the databases in Med Line PubMed, Embase, Cochrane, Ovid-Hinari, Scielo, and Lilacs. Results: 212 articles were reviewed and 58 articles were selected, which were directly related to the topic. GnRH agonists are the only drugs approved by the Food and Drug Administration for reducing the volume of fibroids, used pre-surgically, but have many side effects. The levonorgestrel-releasing device is proposed as an option for patients with fibroids under 6 centimeters and without involvement the uterine cavity. Selective progestin receptor modulators and aromatase inhibitors arise as a management that can be useful, especially for patients in peri-menopause. Conclusions: The literature shows that there is evidence of several drugs that can be used for the management of patients with fibroids especially for the management of associated symptoms to improve quality of life and benefit more patients are those closest to the menopause. Finally there are other drugs that are under investigation and may be future therapeutic options for use both together as myomectomy and exclusive use of medical treatment.

\section{KEY WORDS: Medical management, myoma}




\section{INTRODUCCIÓN}

Los miomas uterinos son los tumores pélvicos femeninos más comunes, con una prevalencia del $20 \%$ al $40 \%$ en edad reproductiva $(1,2)$, sin embargo, su prevalencia puede llegar hasta un $70 \%$ en mujeres blancas y $80 \%$ en mujeres de raza negra (3).

La mayoría de los miomas uterinos son asintomáticos $(1,4)$, en ocasiones pueden producir sangrado, dolor y sensación de presión pélvica, lo que afecta la calidad de vida de las pacientes que los presentan (5), y son la causa más común de histerectomía (2).

La falta de un tratamiento médico eficaz ha hecho que la cirugía sea el manejo tradicional para esta enfermedad (6), con ocasional utilización de las técnicas radiológicas (7).

Algunos avances se han logrado en la aparición y crecimiento de los miomas con el conocimiento de la biología de las células madre, los cuales han permitido que a parte de los factores de riesgo conocidos, (la menarquia temprana, la nuliparidad, la herencia, la obesidad, entre otros), y el papel de los esteroides sexuales se identifiquen factores genéticos y epigenéticos, factores de crecimiento (quimioquinas, citoquinas, y componentes de la matriz glandular), aportes que han permitido mayor conocimiento en el tratamiento médico de estos tumores (8).

En la actualidad, los tratamientos médicos sólo se utilizan a corto plazo, debido a los riesgos significativos con la terapia a largo plazo, o la falta de pruebas con respecto a los beneficios de los nuevos agentes médicos. El tratamiento médico de los miomas es una estrategia reciente. Resulta atractivo para muchos ginecólogos por su facilidad y menores complicaciones cuando se compara con la cirugía, especialmente cuando la principal intención es la preservación de la fertilidad o el deseo de conservar el útero $(9,10)$. Se recomienda terapia con medicamentos para los miomas en las siguientes situaciones (11): i. Como tratamiento sintomático para el alivio temporal de los síntomas por períodos cortos; esta aplicación es adecuada en las mujeres con miomas sintomáticos en la peri-menopausia. ii. En pacientes no aptos para la cirugía por razones médicas. iii. Como un complemento pre-operatorio, para reducir el tamaño de los fibromas, para controlar el sangrado, mejorar los niveles de hemoglobina, o para reducción en el tamaño del mioma, y así cambiar la vía del procedimiento, por ejemplo una histerectomía abdominal por una laparoscópica, o por una histerectomía vaginal sin prolapso (11).

El objetivo de esta revisión es proporcionar un enfoque basado en la calidad de la mejor evidencia disponible de las diferentes opciones de tratamiento médico para los miomas uterinos. Las principales preguntas que se pretenden responder son: ¿qué recursos médicos existen para el manejo de los miomas como tratamiento exclusivo?, ¿cuál es la utilidad de la terapia con medicamentos antes de la cirugía?, ¿cuál es el nivel de evidencia para su uso?

\section{MÉTODOS}

Se realizó una búsqueda bibliográfica en las bases de datos, con la palabras clave, medical therapy, medical management, non-surgical management AND uterine fibroids or myoma or leiomyoma, igualmente, non steroidal anti-inflammatory drugs, hormonal contraceptives or oral contraceptive pills, progestins, progesterone-releasing intrauterine device, selective progesterone-receptor modulators, mifepristone, gonadotropin-releasing hormone agonists, gonadotropin-releasing hormone antagonist, aromatase inhibitors, tranexamic acid, selective estrogen receptor modulators (SERMs), danazol, herbal preparations, acupuncture AND uterine fibroids or myoma or leiomyoma en Med line PubMed, Embase, Cochrane, Ovid-Hinari, Scielo, Bireme y Lilacs. Se revisaron los artículos de los últimos 20 años en idioma inglés, francés, portugués y español. Se seleccionaron estudios clínicos con la mayor evidencia disponible, centrándose en estudios clínicos aleatorizados, revisiones sistemáticas y metaanálisis.

\section{RESULTADOS}

Se revisaron 212 artículos y se seleccionaron 58 , los cuales estaban relacionados directamente con el tema. Es necesario mencionar que según el Colegio Americano de Obstetras y Ginecólogos (ACOG) (3) y las guías francesas en los casos de miomas asintomáticos no hay razón para tratamiento (12). A continuación se mencionan los tratamientos médicos usados y la respectiva evidencia disponible.

Antiinflamatorios (AINES). Estos no han mostrado eficacia en el tratamiento sintomático de los miomas; en un estudio controlado con placebo doble ciego, en 25 mujeres con menorragia, 11 de las cuales también tenían miomas, en el $36 \%$ de las pacientes se presentó disminución de la menorragia idiopática, pero no en quienes tenían miomas (13).

Anticonceptivos orales (ACO). Son considerados de primera línea para el control del sangrado anormal y dismenorrea en pacientes con o sin miomas, sin embargo, estos tratamientos han demostrado utilidad a corto plazo, por que posteriormente la mayoría de las pacientes a las que se recomienda su uso, requieren tratamiento complementario (3).

Progestágenos o dispositivos intrauterinos liberadores de progestágeno. En un estudio descriptivo efectuado en África sobre la eficacia de la medroxiprogesterona, se logró reducción del volumen uterino en un $48 \%$ y del mioma en un $33 \%$, pero el número de pacientes fue muy limitado solo 
veinte pacientes (14); por otra parte, el sistema intrauterino liberador de progestágeno es considerado una efectiva terapia en la hemorragia asociada con miomas, con mejoría de los niveles de hemoglobina, pero se requieren estudios aleatorizados controlados $(15,16,17)$.

Grigorieva y cols (18), en un estudio descriptivo, con el dispositivo liberador de levonorgestrel en 67 pacientes, de los cuales 61 terminaron el seguimiento a los 12 meses, demostraron disminución en el sangrado menstrual para pacientes con miomas que no afectan la cavidad uterina, y con volúmenes uterinos entre $138 \pm 72 \mathrm{ml}$, lograron disminución del volumen en un 18\% (122 $73 \mathrm{ml})$.

En una revisión sistemática, sobre el uso del dispositivo liberador de levonogestrel, Zapata y cols (19), encontraron que no aumenta el sangrado en pacientes con miomas, disminuye la cantidad de flujo menstrual, pero hay un importante número de casos de sangrado irregular y una mayor tasa de expulsión.

El reporte de Sayed y cols, en un estudio aleatorizado controlado, donde comparan el dispositivo liberador de levonogestrel con anticonceptivos orales, se observó una disminución del sangrado en un $91 \%$ a los 12 meses en el grupo levonorgestrel, comparada con el $25 \%$ del grupo de anticonceptivos. Una de las conclusiones de este estudio es que puede ser usado en pacientes seleccionadas con fibromas, que deseen preservar su fertilidad, pero se requieren estudios con un mayor número de pacientes (20).

Respecto a los progestágenos de nueva generación, Ichigo y cols (21), analizaron 55 pacientes con endometriosis que coexistían con miomas a los que se les administró dienogest, encontraron una disminución en el volumen de los miomas del $59,7 \pm 7 \%$, comparado con 12 pacientes que recibieron análogos, los cuales presentaron una disminución del $51,9 \pm 5,5 \%$; lo que permite concluir que el dienogest puede ser usado para mujeres que deseen evitar una cirugía (21). Sin embargo, no hay suficiente evidencia que soporte el uso de progestágeno en pacientes con miomas uterinos (15).

Agonistas de Hormona liberadora de Gonadotrofinas (GnRH). Lethaby y cols (22), en una revisión sistemática con el uso de análogos de $\mathrm{GnRH}$, determinaron que había un aumento preoperatorio en la hemoglobina y el hematocrito, con disminución de la necesidad de incisión mediana, y mayor posibilidad de realizar la cirugía por vía vaginal; por otra parte, encontraron significancia estadística en la disminución de la pérdida sanguínea (58 mililitros) durante la cirugía, cantidad que no tiene significancia clínica. También encontraron como desventaja la posibilidad de recurrencia de los miomas, atribuida que los fibromas pequeños no son observados al momento de la cirugía. Iguales conclusiones se encontraron en la revisión de Cochrane (23).
Igualmente, Vercellini y cols (24), usando análogos tres meses previos a la cirugía, se logró una tasa más alta de histerectomías por vía vaginal $(53 \%)$, comparada con las pacientes llevadas inmediatamente a cirugía(16\%) (24).

Vercellini y cols (25), en un estudio aleatorizado controlado consideran que no se justifica el uso de análogos en pacientes a quienes se va a realizar miomectomía, porque no disminuye la pérdida sanguínea intraoperatoria.

En cuanto al uso de análogos en miomectomía por histeroscopia, Muzii y cols (26), en un estudio aleatorizado multicéntrico en miomas tipo 0 o 1 de acuerdo a la clasificación de la Sociedad Europea de Endoscopia, tamaño entre 10 y 35 mm, encontraron beneficio para disminuir el tiempo de cirugía y la absorción de fluidos. Sin embargo, Mavrelos y cols (27), en otro estudio aleatorizado con 47 pacientes, no demostraron beneficio en el uso de análogos GnRH previos a la cirugía, para la resección de miomas tipo I y II.

Terapia "add back" o terapia coadyuvante. Dado que el uso prolongado de los análogos agonistas de la $\mathrm{GnRH}$ producen menopausia química por hipogonadismo hipogonadotropo, se han introducido la llamada terapia add back, basada en la teoría del umbral estrogénico, que postula la existencia de una respuesta jerárquica de diferentes tejidos hormonodependientes al estradiol, que neutraliza estos efectos, mediante el uso coadyuvante concomitante de estrógenos o progestágenos, solos o combinados, Tibolona o Raloxifeno (28). Según, Palomba y cols (29), en un estudio controlado aleatorizado con placebo cuando al análogo de la $\mathrm{GnRH}$ se le asocia Raloxifeno, la reducción del tamaño es mayor si se compara con el análogo solo, sin diferencia en efectos clínicos o de laboratorio (29).

Antagonistas de la Hormona liberadora de Gonadotrofinas (GnRH). En un estudio prospectivo realizado por Flierman y cols (30), con un antagonista de la $\mathrm{GnRH}$ (ganirelix acetato) en 19 pacientes, demostró disminución del $42,7 \%$ en el tamaño de los miomas, y $46,6 \%$ en el tamaño uterino. El elagolix de administración oral $(27,31)$, es otro antagonista promisorio. Estos fármacos requieren estudios aleatorizados controlados para recomendar su uso.

Moduladores selectivos de receptores de progestágeno (SPRMs). La mifepristona ha sido el primero de los SPRMs (1) con el cual se ha demostrado disminución en el tamaño de los miomas. En varios estudios con dosis de 5-50 miligramos por 3 a 6 meses, muestra reducción del 26 al $74 \%$ del volumen y disminución de los síntomas, pero con alta prevalencia de hiperplasia endometrial como efecto secundario. En el uso de la mifepristona hay 3 desventajas (32). Un aumento del 3 al $9 \%$ de las transaminasas, pero nunca por encima de 100 unidades por litro, oleadas de calor del 10 al $20 \%$ y engrosamiento endometrial. 
La revisión sistemática de Steinauer y cols (33), en 2004, mostraron que la mifepristona es efectiva en el tratamiento de los miomas uterinos, pero produce hiperplasia endometrial como efecto secundario. Sin embargo, el estudio de Tristan y cols (34), en su revisión de Cochrane concluyen que la mifepristona disminuye la hemorragia menstrual y mejora la calidad de vida, pero no disminuye el volumen de los miomas (34).

El metaanálisis de Shen y cols (35), que recolectó 780 mujeres de 11 estudios aleatorizados controlados, se concluye que la mifepristona disminuye significativamente el volumen de los miomas y del útero, con mejoría de los síntomas. La dosis óptima es de 2,5 miligramos por 3 a 6 meses. También llegan a la conclusión en que no hay suficiente evidencia que el tratamiento con este medicamento lleve a hiperplasia endometrial de alto grado.

Más recientemente Yerushalmi y cols (36), reportan el uso de mifepristona por vía vaginal a dosis de 10 miligramos en 33 pacientes, en quienes se logró la disminución del volumen uterino de $135,3 \pm 22,9 \mathrm{~cm}^{3}$ a $101,2 \pm 22 \mathrm{~cm}^{3}$, con disminución en la hemorragia uterina anormal y mejoría de la calidad de vida. No encontraron desarrollo de hiperplasia endometrial.

En la Tabla I se resumen los principales trabajos que comparan la mifepristona con placebo; se describe el número de pacientes que terminaron el estudio, el tiempo usado, la disminución del volumen del mioma y del útero y el porcentaje de hiperplasia (5,37-39). En la Tabla II se comparan diferentes dosis de mifepristona y su eficacia en la reducción del volumen del mioma y del útero y el porcentaje de desarrollo de hiperplasia $(6,32,40-45)$.

En un estudio realizado por Chwalisz y cols (46), con 129 pacientes, con asoprisnil un SPRMs selectivo, las cuales fueron aleatorizados en cuatro grupos, con dosis de 5, 10 y 25 miligramos y placebo, demostraron eficacia en la mejoría de los síntomas, disminución del sangrado, disminución del volumen uterino y del mioma dominante; efectos más evidentes con dosis de 25 miligramos, pero encontraron oleadas de calor entre 3 y $10 \%$ en las diferentes dosis y formación de quistes funcionales en los ovarios. Desafortunadamente, no se hizo seguimiento posterior a la suspensión del medicamento, se requieren más estudios para recomendar su uso.

El acetato ulipristal (VA2914 CDD2914), es uno de los SPRMs selectivos, que induce apoptosis de las células del mioma en vitro $(4,47)$. Por otro lado, Williams y cols (48), demostraron que después del tratamiento con acetato ulipristal, el epitelio glandular parecía inactivo; se presentó un leve engrosamiento reversible del endometrio en una minoría de casos. Donnez y cols (2), en un estudio fase III, aleatorizaron tres grupos, con dosis de 5 y 10 miligramos de ulipristal y un tercer grupo con placebo por 13 semanas; encontraron disminución significativa del volumen del mioma y de la hemorragia en ambas dosis, respecto al placebo. En otro estudio, también realizado por Donnez y cols (7), compararon el ulipristal con leuprolide en 290 pacientes, con dosis de ulipristal de $5 \mathrm{mg}$ y $10 \mathrm{mg}$ en 100 y 95 pacientes respectivamente, y leuprolide a dosis de 3,75 mg en 95 mujeres; se logró reducción del volumen de los miomas del $36 \%$ al $42 \%$ con ulipristal, y del $53 \%$ con leuprolide; los síntomas vasomotores afectaron al $10-11 \%$ de las pacientes del primer medicamento y al $40 \%$ del grupo de leuprolide; aproximadamente la mitad de las pacientes fueron llevadas a cirugía y el otro $50 \%$ fue seguido a 6 meses. No se presentó diferencias en la mejoría de la calidad de vida, y encontraron que al mes se empezó a presentar aumento en el tamaño de los miomas de las pacientes con acetato leuprolide y la reducción del volumen se mantuvo por más tiempo en el grupo de ulipristal.

\section{Tabla I \\ EFECTO SOBRE EL VOLUMEN DEL MIOMA Y DEL ÚTERO, CON EL USO DE LA MIFEPRISTONA VS PLACEBO}

\begin{tabular}{lccccccc}
\hline Autores & $\begin{array}{c}\text { Dosis vs } \\
\text { placebo }\end{array}$ & $\begin{array}{c}\text { Pacientes que } \\
\text { terminan el } \\
\text { estudio }\end{array}$ & $\begin{array}{c}\text { Tiempo } \\
\text { (meses) }\end{array}$ & $\begin{array}{c}\text { Amenorrea } \\
(\%)\end{array}$ & $\begin{array}{c}\text { Reducción } \\
\text { volumen } \\
\text { uterino (\%) }\end{array}$ & $\begin{array}{c}\text { Reducción } \\
\text { volumen del } \\
\text { mioma (\%) }\end{array}$ & $\begin{array}{c}\text { Hiperplasia } \\
\text { endometrial } \\
(\%)\end{array}$ \\
\hline Fiscella & $5 \mathrm{mg}$ & 22 & 6 & $\mathrm{NR}$ & 47 & $\mathrm{NR}$ & 0 \\
$(2006$, EEUU) & placebo & 20 & 6 & $\mathrm{NR}$ & 0 & $\mathrm{NR}$ & 0 \\
Engman & $50 \mathrm{mg}$ & 14 & 3 & $\mathrm{NR}$ & $\mathrm{NR}$ & 27 & 0 \\
$(2009$, Suecia) & placebo & 14 & 3 & $\mathrm{NR}$ & $\mathrm{NR}$ & 0 & 0 \\
Bagaria & $10 \mathrm{mg}$ & 19 & 3 & 84,2 & 26,6 & 30 & 63,1 \\
(2009, India) & placebo & 16 & 3 & 0 & 0 & 0 & 0 \\
Carbonell & $5 \mathrm{mg}$ & 47 & 3 & 93,1 & $\mathrm{NR}$ & 28,5 & 0 \\
(2013, Cuba) & placebo & 58 & 3 & 4,3 & $\mathrm{NR}$ & 0 & 0 \\
\hline
\end{tabular}

NR: No hay reporte. 


\section{Tabla II \\ EFECTO SOBRE EL VOLUMEN DEL MIOMAY DEL ÚTERO CON LAS DIFERENTES DOSIS DE MIFEPRISTONA Y TIEMPO DE TRATAMIENTO}

\begin{tabular}{|c|c|c|c|c|c|c|c|}
\hline Autores & $\begin{array}{l}\text { Dosis } \\
(\mathrm{mg})\end{array}$ & $\begin{array}{l}\text { Pacientes } \\
\text { (n) }\end{array}$ & $\begin{array}{c}\text { Tiempo de } \\
\text { tratamiento } \\
\text { (meses) }\end{array}$ & $\begin{array}{l}\text { Pacientes con } \\
\text { amenorrea (\%) }\end{array}$ & $\begin{array}{l}\text { Reducción } \\
\text { volumen del } \\
\text { mioma (\%) }\end{array}$ & $\begin{array}{c}\text { Reducción } \\
\text { volumen } \\
\text { uterino (\%) }\end{array}$ & $\begin{array}{l}\text { Hiperplasia } \\
(\%)\end{array}$ \\
\hline Eisinger & 5 & 14 & 6 & 61 & NR & 48 & 25 \\
\hline (2003, EEUU) & 10 & 20 & 6 & 65 & NR & 49 & 30 \\
\hline $\begin{array}{l}\text { Carbonell } \\
(2008, \text { Cuba) }\end{array}$ & 5 & 49 & 3 & 89,8 & 45 & 36 & 0 \\
\hline $\begin{array}{l}\text { Carbonell } \\
\text { (2010, Cuba) }\end{array}$ & $\begin{array}{c}5 \\
10\end{array}$ & $\begin{array}{l}48 \\
52\end{array}$ & $\begin{array}{l}3 \\
3\end{array}$ & $\begin{array}{l}95,8 \\
86,5\end{array}$ & $\begin{array}{l}47,5 \\
38,3\end{array}$ & $\begin{array}{c}27 \\
25,1\end{array}$ & $\begin{array}{l}0 \\
0\end{array}$ \\
\hline $\begin{array}{l}\text { Carbonell } \\
\text { (2010, Nicaragua) }\end{array}$ & $\begin{array}{c}5 \\
10\end{array}$ & $\begin{array}{l}44 \\
43\end{array}$ & $\begin{array}{l}3 \\
3\end{array}$ & $\begin{array}{c}86,4 \\
93\end{array}$ & $\begin{array}{l}60,8 \\
59,4\end{array}$ & $\begin{array}{l}34,4 \\
30,4\end{array}$ & $\begin{array}{l}8,8^{\star} \\
8,8^{\star}\end{array}$ \\
\hline $\begin{array}{l}\text { Carbonell } \\
(2012, \text { Cuba) }\end{array}$ & $\begin{array}{c}5 \\
10\end{array}$ & $\begin{array}{l}82 \\
78\end{array}$ & $\begin{array}{l}6 \\
6\end{array}$ & $\begin{array}{l}42 \\
86\end{array}$ & $\begin{array}{l}39,1 \\
48,1\end{array}$ & $\begin{array}{l}27,2 \\
30,3\end{array}$ & $\begin{array}{l}2,4 \\
1,3\end{array}$ \\
\hline $\begin{array}{l}\text { Carbonell } \\
(2013, \text { Cuba) }\end{array}$ & $\begin{array}{c}5 \\
10\end{array}$ & $\begin{array}{l}35 \\
35\end{array}$ & $\begin{array}{l}9 \\
9\end{array}$ & $\begin{array}{l}82,1 \\
100\end{array}$ & $\begin{array}{l}65,8 \\
52,2\end{array}$ & $\begin{array}{l}33,4 \\
38,5\end{array}$ & $\begin{array}{l}0 \\
0\end{array}$ \\
\hline $\begin{array}{l}\text { Carbonell } \\
(2013, \text { Cuba) }\end{array}$ & $\begin{array}{c}2,5 \\
5\end{array}$ & $\begin{array}{l}102 \\
106\end{array}$ & $\begin{array}{l}3 \\
3\end{array}$ & $\begin{array}{c}78 \\
93,6\end{array}$ & $\begin{array}{l}27,9 \\
45,5\end{array}$ & $\begin{array}{l}18,2 \\
22,1\end{array}$ & $\begin{array}{l}0 \\
0\end{array}$ \\
\hline $\begin{array}{l}\text { Kulshrestha } \\
(2013 \text {, India) }\end{array}$ & $\begin{array}{l}10 \\
25\end{array}$ & $\begin{array}{l}73 \\
70\end{array}$ & $\begin{array}{l}3 \\
3\end{array}$ & $\begin{array}{l}90,4 \\
95,7\end{array}$ & $\begin{array}{l}22,5 \\
35,7\end{array}$ & $\begin{array}{l}\text { NR } \\
\text { NR }\end{array}$ & $\begin{array}{l}6,9 \\
9,4\end{array}$ \\
\hline
\end{tabular}

* Posterior a nuevo análisis 0 por ciento. NR: No hay reporte.

El proellex (CDB4124), es otro de los SPRMs selectivo que ha demostrado disminución en el tamaño de los miomas (8) pero requiere estudios aleatorizados controlados.

Inhibidores de aromatasa. Hilario y cols (49), encontraron que con el uso de anastrazol se bloquea la síntesis de estrógenos, con lo cual se logra reducción del volumen del útero entre el 9-32\%, con mejoría de los síntomas y sin cambios de hipoestrogenismo. La investigación de Parsanezhad y cols (10), en un estudio aleatorizado controlado multicéntrico encontraron ventajas cuando se comparó un inhibidor de la aromatasa versus un análogo de la $\mathrm{GnRH}$. Los hallazgos favorecen a los inhibidores de la aromatasa respecto a sus menores efectos secundarios y mayor reducción del volumen uterino (45,6 vs $33,2 \%)(10)$.

Ácido tranexámico. Este fármaco es un bloqueador del punto de enlace de la lisina en la enzima plasmina, esencial para el enlace de la plasmina con la fibrina. En un estudio realizado por Caglar y cols (50), con 100 pacientes, a quienes se les realizó miomectomía y se les administró ácido tranexámico por vía endovenosa, no se demostró beneficio en la disminución de la pérdida sanguínea, y no existe evidencia para su recomendación (50).
Moduladores selectivos de receptores estrogénicos (SERMS): Jirecek y cols (51), usando Raloxifeno de 180 miligramos al día encontraron prevención en la progresión del crecimiento de los miomas comparado con el grupo control sin medicación y resultó una disminución en el volumen de los miomas en un $9,1 \%$ comparado con el inicio y un $22,2 \%$ comparado con el control sin significancia estadística. Por otra parte, Sadan y cols (52), en un estudio con tamoxifeno consideraron que no se debe usar por los efectos secundarios. No hay evidencia suficiente para recomendar el uso de los moduladores selectivos de los receptores estrogénicos (SERMS) en disminución del tamaño o mejoría de los síntomas (53).

Danazol. Respecto a este medicamento a la fecha de la publicación no existen estudios aleatorizados controlados que soporten que los beneficios sean mayores que los riesgos para el tratamiento de los miomas sintomáticos (54).

Preparaciones a base de hierbas. Roshdy y cols (55), en un estudio clínico aleatorizado controlado con placebo en 33 pacientes, encontraron disminución en el volumen de los miomas en un $32,6 \%$ en pacientes que recibieron extracto de té verde por 4 meses, comparado con el placebo, sin embargo, el 
número de pacientes no permite sacar conclusiones y se requieren estudios para apoyar o rechazar el uso en esta enfermedad (56).

Acupuntura. El uso de la acupuntura en el tratamiento de los miomas es incierto, se requieren estudios aleatorizados controlados, acerca de su eficacia y seguridad (57).

Otros medicamentos. Pourmatroud y cols (58), usaron vitamina $C$ de $2 \mathrm{~g}$ durante la miomectomía en 102 pacientes, encontraron disminución en la pérdida sanguínea, cuando se usaba concomitante durante la cirugía. Otros productos farmacológicos han sido objeto de investigación en el tratamiento médico de los miomas, como los moduladores selectivos de receptores progestacionales CP8863 y CP8947, la rosiglitazona y ciglitizona, la pirfenidona, una molécula de piridina con efecto antifibrótico, ha sido también usada por su efecto inhibidor de la proliferación de las células miometriales y del mioma in vitro $(8,11)$. El tranilast, los derivados del ácido retinoico, la cabergolina, los derivados de heparina, el interferón-a, los análogos de la somastatina, el isoliquiritigenin, la corcumina y la vitamina $D$, no presentan resultados contundentes $(8,28)$.

\section{CONCLUSIONES}

La literatura muestra que hay evidencia de varios medicamentos que pueden ser usados para el manejo de pacientes con miomatosis especialmente para el manejo de los síntomas asociados para mejorar su calidad de vida, las pacientes que obtienen mayor beneficio son las que están cercanas a la menopausia. Finalmente hay otros fármacos que son objeto de investigación y pueden ser futuras opciones terapéuticas para su uso en conjunto tanto en la miomectomía y como uso exclusivo de tratamiento médico.

DECLARACIÓN: Parte de esta revisión fue presentada como ponencia en el XII Congreso Sociedad Iberoamericana de Endoscopia Ginecológica (SIAEGI) y III Congreso Colombiano de Endoscopia Ginecológica. Cartagena, Colombia 25 de Noviembre de 2013.

\section{REFERENCIAS}

1. Tropeano G, Amoroso S, Scambia G. Non-surgical management of uterine fibroids. Human Reproduction Update 2008;14(3): 259-274

2. Donnez J, Tatarchuk TF, Bouchard P, Puscasiu L, Zakharenko NF, Ivanova T, et al. Ulipristal Acetate versus Placebo for Fibroid Treatment before Surgery. N Engl J Med 2012;366:409-20.

3. ACOG Practice Bulletin. Alternatives to Hysterectomy in the Management of Leiomyomas. Clinical Management Guidelines for Obstetrician-Gynecologists 2008; 112(2). PART 1.

4. Croxtall JD. Ulipristal Acetate In Uterine Fibroids. Drugs 2012;72 (8):1075-1035

5. Engman M, Granberg S, Williams ARW, Meng CX,
Lalitkumar PGL, Gemzell-Danielsson K. Mifepristone for treatment of uterine leiomyoma. A prospective randomized placebo controlled trial. Human Reproduction 2009;24(8):1870-9.

6. Carbonell Esteve JL, Acosta R, Heredia B, Pérez Y, Yero Castañeda MC, Seigler I, Andreu Ballester JC. Mifepristona para tratamiento del fibroma uterino. Rev Cubana Obstet Ginecol 2010;36(2)130-44.

7. Donnez J, Tomaszewski J, Vázquez F, Bouchard P, Lemieszczuk B, Baró F, et al. Ulipristal Acetate versus Leuprolide Acetate for Uterine Fibroids. N Engl J Med 2012;366:421-32.

8. Islam S, Protic O, Giannubilo SR, Toti P, Tranquilli AL, Petraglia F, Castellucci M, et al. Uterine Leiomyoma: Available medical treatments and new possible therapeutic options. J Clin Endocrinol Metab 2013;98:92134.

9. Lu D, Lu X, Navaratnam K, Shi G. Aromatase inhibitors for uterine fibroids. Cochrane Database of Systematic Reviews 2011;(12):CD009505.

10. Parsanezhad ME, Azmoon M, Alborzi S, Rajaeefard A, Zarei A, Kazerooni T, Frank V, Schmidt EH. A randomized, controlled clinical trial comparing the effects of aromatase inhibitor (letrozole) and gonadotropinreleasing hormone agonist (triptorelin) on uterine leiomyoma volume and hormonal status. Fertil Steril 2010;93:192-8.

11. Sankaran S, Manyonda IT. Medical management of fibroids. Best Pract Res Clin Obstet Gynaecol 2008; 22(4):655-76.

12. Marret H, Fritel X, Ouldamer L, Bendifallah S, Brun JL, De Jesus I, et al. Therapeutic management of uterine fibroid tumors: updated French guidelines. Eur J ObstetGynecol Reprod Biol 2012;165:156-64.

13. Parker WH. Uterine myomas: management. Fertil Steril 2007;88:255-71.

14. Venkatachalam S, Bagratee JS, MoodleyJ. Medical management of uterine fibroids with medroxy progesterone acetate (Depo Provera): a pilot study. J Obstet Gynaecol 2004;24( 7):798-800.

15. Soysal S, Soysal ME. The efficacy of levonorgestrelreleasing intrauterine device in selected cases of myoma-related menorrhagia: a prospective controlled trial. Gynecol Obstet Invest 2005;59:29-35.

16. Sangkomkamhang US, Lumbiganon $P$, Laopaiboon $\mathrm{M}$, Mol BWJ. Progestogens or progestogen-releasing intrauterine systems for uterine fibroids. Cochrane Database of Systematic Reviews 2013;(2):CD008994.

17. Gunes M, Ozdegirmenci O, Kayikcioglu F, Haberal A, Kaplan M. The effect of levonorgestrel intrauterine system on uterine myomas: A 1-year follow-up study. $\mathrm{J}$ Min Inv Gynecol 2008;15:735-8.

18. Grigorieva V, Chen-Mok M, Tarasova M, Mikhailov A. Use of a levonorgestrel-releasing intrauterine system to treat bleeding related to uterine leiomyomas. Fertil Steril 2003;79:1194-8.

19. Zapata LB, Whiteman MK, Tepper NK, Jamieson DJ, Marchbanks PA, Curtis KM. Intrauterine device use among women with uterine fibroids: a systematic review. Contraception 2010;82:41-55.

20. Sayed GH, Zakherah MS, El-Nashar SA, Shaaban MM. A randomized clinical trial of a levonorgestrelreleasing intrauterine system and a low-dose combined oral contraceptive for fibroid-related menorrhagia. Inter J Gynecol Obstet 2011;112:126-30.

21. Ichigo S, Takagi H, Matsunami K, Suzuki N, Imai A. 
Beneficial effects of dienogest on uterine myoma volume: a retrospective controlled study comparing with gonadotropin-releasing hormone agonist. Arch Gynecol Obstet 2011;284:667-70.

22. Lethaby A, Vollenhoven B, Sowter M. Efficacy of pre-operative gonadotrophin hormone releasing analogues for women with uterine fibroids undergoing hysterectomy or myomectomy: a systematic review. BJOG 2002;109:1097-108.

23. Lethaby A, Vollenhoven B, Sowter MC. Pre-operative $\mathrm{GnRH}$ analogue therapy before hysterectomy or myomectomy for uterine fibroids. Cochrane Database of Systematic Reviews 2001;(2):CD000547.

24. Vercellini P. Crosignani PG, Mangioni C, Imparato E, Ferrari A, De Giorgi O. Treatment with a gonadotrophin releasing hormone agonist before hysterectomy for leiomyomas: results of a multicentre, randomised controlled trial. Br J Obstet Gynaecol 1998;105:114854.

25. Vercellin P, Trespıdi L, Zaina B, Vicentini S, Stellato G, Crosignani PG. Gonadotropin-releasing hormone agonist treatment before abdominal myomectomy: a controlled trial. Fertil Steril 2003;79:1390-5.

26. Muzii L, Boni T, Bellati F, Marana R, Ruggiero A, Zullo MA, Angioli R, PaniciPB. GnRH analogue treatment before hysteroscopic resection of submucous myomas: a prospective, randomized, multicenter study. Fertil Steril 2010;94:1496-9.

27. Mavrelos D, Ben-Nagi J, Davies A, Lee C, Salim R, Jurkovic $D$. The value of pre-operative treatment with $\mathrm{GnRH}$ analogues in women with submucous fibroids: a double-blind, placebo-controlled randomized trial. Hum Reprod 2010;25(9):2264-69.

28. Sabry M, Al-Hendy A. Medical Treatment of Uterine Leiomyoma. Reprod Sci 2012;19(4) 339-53.

29. Palomba S, Russo T, Orio Jr F, TauchmanovaL, Zupi E, Benedetti Panici PL, et al. Effectiveness of combined $\mathrm{GnRH}$ analogue plus raloxifene administration in the treatment of uterine leiomyomas: a prospective, randomized, single-blind, placebo-controlled clinical trial. Hum Reprod 2002;17(12):3213-19.

30. Flierman PA, Oberye JJL, van der Hulst VPM, de Blok S. Rapid reduction of leiomyoma volume during treatment with the $\mathrm{GnRH}$ antagonist ganirelix. BJOG 2005;112:638-42.

31. Struthers RS, Nicholls AJ, Grundy J, Chen T, Jimenez R, Yen SS, et al. Suppression of Gonadotropins and Estradiol in Premenopausal Women by Oral Administration of the Nonpeptide Gonadotropin-Releasing Hormone Antagonist Elagolix. J Clin Endocrinol Metab 2009;94(2):545-51.

32. Carbonell JL, Acosta R, Pérez $Y$, Marrero AG, Trellez E, Sánchez C, Tomasi G. Safety and effectiveness of different dosage of mifepristone for the treatment of uterine fibroids: a double-blind randomized clinical trial. Int J Women's Health 2013;5:115:24.

33. Steinauer J, Pritts EA, Jackson R, Jacoby AF.Systematic Review of mifepristone for the treatment of uterine leiomyomata. Obstet Gynecol 2004;103:1331-6.

34. Tristan M, Orozco LJ, Steed A, Ramírez- Morera A, Stone $\mathrm{P}$. Mifepristone for uterine fibroids. Cochrane Database of Systematic Reviews 2012;(8):CD007687.

35. Shen Q, Hua Y, Jiang W, Zhang W, Chen M, Zhu X. Effects of mifepristone on uterine leiomyoma in pre- menopausal women: a meta-analysis. Fertil Steril 2013;100(6):1722-6.

36. Yerushalmi GM, Gilboa Y, Jakobson-Setton A, Ta$\operatorname{dir}$ Y, Goldchmit C, Katz D, Seidman DS. Vaginal mifepristone for the treatment of symptomatic uterine leiomyomata: an open-label study. Fertil Steril 2014;101(2):496-500.

37. Fiscella K, Eisinger SH, Meldrum S, FengCh, Fisher SG, Guzick, DS. Effect of mifepristone for symptomatic leiomyomata on quality of life and uterine size. A randomized controlled trial. Obstet Gynecol 2006;108:1381-7.

38. Bagaria M, Suneja A, Vaid NB, Guleria K, Mishra K. Low-dose mifepristone in treatment of uterine leiomyoma: A randomised double-blind placebo-controlled clinical trial. Aust N Z J Obstet Gynecol 2009;49:7783.

39. Carbonell JL, Acosta R, Pérez Y, Rodriguez B, Seigler I, Sánchez C, Tomasi G. Mifepristone versus placebo to treat uterine myoma: a double-blind, randomized clinical trial. Int J Women's Health 2013;5:361-9.

40. Eisinger SH, Meldrum S, Fiscella K, le Roux HD, Guzick DS. Low-dose mifepristone for uterine leiomyomata. Obstet Gynecol 2003;101(2):243-50.

41. Carbonell JL, Acosta R, Heredia B, Pérez Y, Yero Castañeda M, Valle Hernández A. Mifepristone for the treatment of uterine leiomyomas. A randomized controlled trial. Obstet Gynecol 2008;112:1029-36.

42. Carbonell JL, Quiróz GM, Borge A, Castellón Zapata LE, Cuadra Aragón W, Tomasi G. Mifepristona 5 mg frente a $10 \mathrm{mg}$ diarios en el tratamiento del leiomioma. Ensayo clínico aleatorizado. Prog Obstet Ginecol 2010;53(2):51-8.

43. Carbonell JL, Acosta R, Pérez Y, Campos R, Valle A, Sanchez C. Treatment of uterine myoma with 5 or 10 mg mifepristone daily during 6 months, post-treatment evolution over 12 months: double-blind randomised clinical trial. Eur J Obstet Gynecol Reprod Biol 2012;161:202-8.

44. Carbonell JL, Acosta R, Pérez Y, Garcés R, Sánchez $\mathrm{C}$, Tomasi G. Clinical Study. Treatment of uterine myoma with 2.5 or $5 \mathrm{mg}$ mifepristone daily during 3 months with 9 months posttreatment followup: randomized clinical trial. ISRN Obstet Gynecol 2013;64930:1-8.

45. Kulshrestha V, Kiriplani A, Agarwal N, Sareen N, Garg $\mathrm{P}$, Hari $\mathrm{S}$ et al. Low dose mifepristone in medical management of uterine leiomyoma-an experience from a tertiary care hospital from north India. Indian J Med Res 2013;137:1154-62.

46. Chwalisz K, Larsen L, Mattia-Goldberg C, Edmonds A, Elger W, Winkel CA. A randomized, controlled trial of asoprisnil, a novel selective progesterone receptor modulator, in women with uterine leiomyomata. Fertil Steril 2007;87(6):1399-412.

47. Biglia N, Carinelli S, Maiorana A, D'Alonzo M, Lo Monte G, Marci R. Ulipristal acetate: a novel pharmacological approach for the treatment of uterine fibroids. Drug Des Devel Ther 2014;8:285-92.

48. Williams ARW, Bergeron C, Barlow DH, Ferenczy A. Endometrial morphology after treatment of uterine fibroids with the selective progesterone receptor modulator, ulipristal acetate. Int J Gynecol Pathol 2012;31:556-69.

49. Hilario SG, Bozzini N, Borsari R, Baracat EC. Action of aromatase inhibitor for treatment of uterine 
leiomyoma in perimenopausal patients. Fertil Steril 2009;91(1):240-3.

50. Caglar GS, Tasci Y, Kayikcioglu F, Haberal A. Intravenous tranexamic acid use in myomectomy: A prospective randomized double-blind placebo controlled study. Eur J Obstet Gynecol Reprod Biol 2008;137:227-31.

51. Jirecek S, Lee A, Pavo I, Crans G, Eppel W, Wenzl R. Raloxifene prevents the growth of uterine leiomyomas in premenopausal women. Fertil Steril 2004;81:132-6.

52. Sadan O, Ginath S, Sofer D, Rotmensch S, Debby $A$, Glezerman M. et al. The role of tamoxifen in the treatment of symptomatic uterine leiomyomata - a pilot study. Eur J Obstet Gynecol Reprod Biol 2001;96:183-6.

53. Deng L, Wu T, Chen XY, Xie L, Yang J. Selective estrogen receptor modulators (SERMs) for uterine leiomyomas. Cochrane Database of Systematic Reviews 2012;(10):CD005287.
54. Ke LQ, Yang K, Li CM, Li J. Danazol for uterine fibroids. Cochrane Database of Systematic Reviews 2009; ( 3):CD007692.

55. Roshdy E, Rajaratnam V, Maitra S, Sabry M, Ait Allah AS, Al-endy A.Treatment of symptomatic uterine fibroids with green tea extract: a pilot randomized controlled clinical study. Int J Women's Health 2013;5:477-86.

56. Liu JP, Yang H, Xia Y, Cardini F. Herbal preparations for uterine fibroids. Cochrane Database of Systematic Reviews 2013;(4):CD005292.

57. Zhang Y, Peng W, Clarke J, Zhishun L. Acupuncture for uterine fibroids. Cochrane Database of Systematic Reviews 2010;(1):CD007221.

58. Pourmatroud E, Hormozi L, Hemadi M, Golshahi R. Intravenous ascorbic acid (vitamin $\mathrm{C}$ ) administration in myomectomy: a prospective, randomized, clinical trial. Arch Gynecol Obstet 2012;285:111-5. 